\title{
Application of Haptic Feedback to a Combot
}

\author{
Samuel McAmis* and Kyle B. Reed ${ }^{\dagger}$ \\ Department of Mechanical Engineering \\ University of South Florida \\ Tampa, Florida, 33612
}

\begin{abstract}
In the sport of combat robotics, there is a strong incentive to produce machines with high power-to-weight ratios. To produce more power, electric motors in these machines are operated well above their rated specifications, leading to a shortened working life and the risk of over-temperature failure. This paper discusses the design of a system to haptically display the instantaneous current draw and temperature of the motors of a combot and the application of this system to the drivetrain of a preexisting combot. To give the driver a sense of the remotely controlled machine's state, the current draw is presented to the operator by vibrating the control joystick and the temperatures of the handles are controlled to match the temperatures of the motors. Tested in combat, this system shows promise as a means to reduce damage, improve performance, and increase driver awareness of the machine's state.
\end{abstract}

Keywords: Combot, Battlebot, combat robotics, haptics, thermal feedback, vibrotactile feedback

\section{INTRODUCTION}

Started in 1994 [13], the sport of robotic combat pits teleoperated machines against one another within bullet-proof glass arenas with the objective of incapacitating the opponent's machine through physical damage. These machines are typically referred to as "battlebots" or "combots". With the combots divided by weight class, there is a strong incentive to produce machines with high power-to-weight ratios. This incentive leads to designs that push the limits of the motors used. One of the most common failures in these telerobots, not attributable to direct damage, is the melting of internal insulation within motors and the subsequent short circuit and failure of armature windings. If the current draw and temperature of these motors were monitored and displayed to the operator(s), they would have a better idea of whether it is best to continue to push the motors or to be more conservative. However, it is difficult to convey information to the operator without causing a distraction from the movement of the opponent's machine. Haptically rendering the current and temperature information allows the operator to make judgements regarding the condition of the motors without distracting him from events inside the arena.

\section{BACKGROUND}

Typically viewed as a hobby, the combot literature is extremely sparse. Most of the work in this field involves the use of the combot design process as an educational tool for undergraduate engineering classes [10][4][3]. Though there are a handful of books dealing with the topic, the most comprehensive being [8][9], much of the material in this section comes from first-hand experience.

The Robot Fighting League sanctions combat events throughout the year and recognizes weight classes ranging from $150 \mathrm{~g}(5.3 \mathrm{oz})$

\footnotetext{
*e-mail: smcamis@mail.usf.edu

†e-mail: kylereed@usf.edu
}

to $100 \mathrm{~kg}(220 \mathrm{lbs})$. Matches at these events typically last three minutes (two minutes for smaller weight classes). The individual designs of combat robots in all weight classes vary wildly but can be broken down into five general types: horizontal and vertical spinners, flippers, lifters, and wedges. While the first three types utilize high energy weapon systems to attempt to damage their opponent by tearing through their armor or hurling them through the air, the other two groups follow a more conservative approach of using a lighter weapon system in favor of heavier armor and a more maneuverable drivetrain. Common to all designs, however, is a desire to maximize the power to weight ratio of all aspects of the combot. This desire has lead to the popularization of titanium and aerospace grades of aluminum and steel in the frame and armor construction, as well as the use of lithium based battery technologies.

To obtain more power from their motors, competitors typically gear motors for peak power and operate them at up to twice their rated voltage. The heavy loading leads to the motors operating far from their maximum efficiency range, therefore increasing excess heat generation. This can be compounded if there is damage to the combot. In this paper, the authors propose using haptics to convey information about the performance of the motors back to the operator.

This system was implemented on the author's heavyweight ( $220 \mathrm{lb}$ class) combot 'Gruff', shown in Fig. 1, which competed previously at the Combots Cup V. Gruff is a low profile electric lifter with a six wheel drivetrain; some of its other features are listed in Table 1. Unlike most combots, Gruff is controlled by an Innovation First Control System based on a Microchip PIC processor.

Table 1: Features of the combot 'Gruff'

Weight: $218 \mathrm{lb}(99 \mathrm{~kg})$

Dimensions: 38 "x38"x10" (97 cm x $97 \mathrm{~cm}$ x $25 \mathrm{~cm}$ )

Front Armor: $3 / 8$ " $(1 \mathrm{~cm})$ High Strength Steel

Top Speed: $\quad 17 \mathrm{ft} / \mathrm{s}(5 \mathrm{~m} / \mathrm{s})$

Acceleration: $\quad 0$ to $10 \mathrm{ft} / \mathrm{s}(3 \mathrm{~m} / \mathrm{s})$ in $0.8 \mathrm{~s}$

Flipper Lifting Force: $300 \mathrm{lb}(1335 \mathrm{~N})$

Flipper Speed: $\quad 180^{\circ}$ rotation in $0.5 \mathrm{~s}$

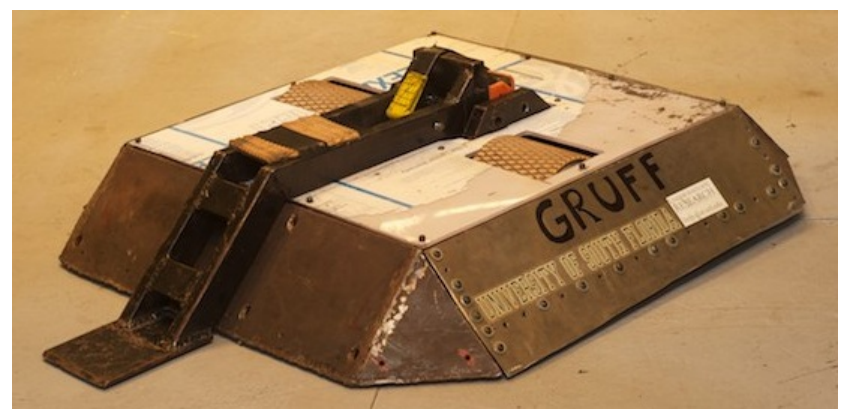

Figure 1: The combot 'Gruff'. 


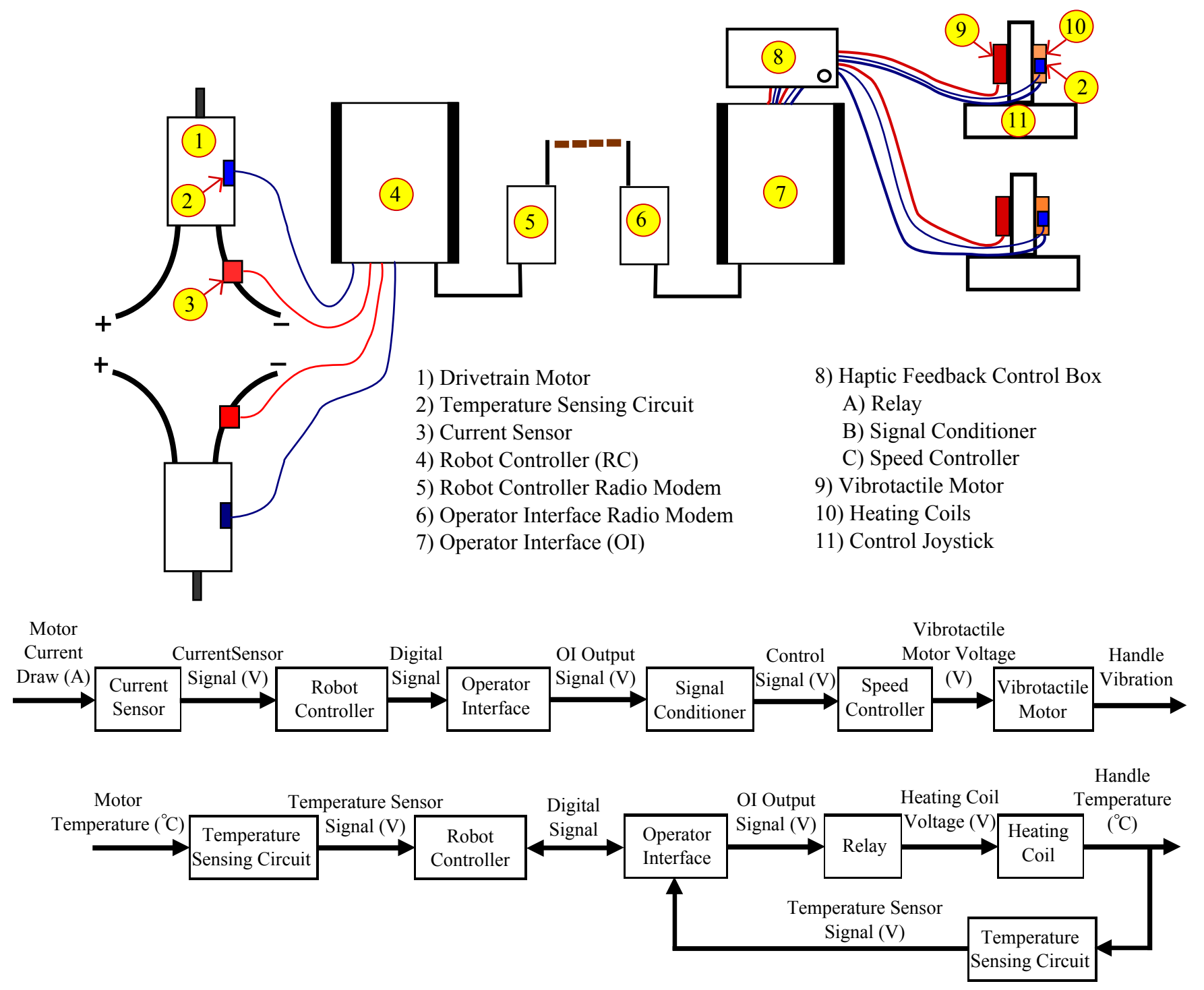

Figure 2: Haptic feedback system diagram (top) and haptic feedback system block diagrams for current (middle) and temperature (bottom).

Traditionally, force feedback has been used to improve performance in teleoperator tasks. Vibration as a representation of force may be used as an alternative to traditional force feedback when a low cost method of feedback is desired or there are concerns about impeding the operator's input [12][7]. Though not as beneficial as traditional force feedback, this method has been validated in virtual interactions [2] and the control of hand prostheses [12]. We believe vibrotactile feedback is best for this application due to the ease of application to the control system and its ability to convey the current draw, related directly to the force exerted by the motors, without interfering with the operator's ability to control the combot.

The importance of thermotactile feedback in teleoperator systems is typically related to the identification of objects by thermal conductivity or the desire to avoid damage to the telerobot from extreme temperatures in the remote environment [1][6]. This application is related to the latter, however, rather than conveying information about possible damage to the end effector, this system is intended to help the operator determine the probability of over-temperature damage to the telerobot's internal components.
The authors are not aware of any previous work on the use of thermotactile feedback for use in combat robotics. Although there is thermal feedback for temperature and vibration feedback for the current draw, mechanoreceptors and thermoreceptors and their afferent pathways are distinct, therefore it is not expected that the vibrotactile and thermotactile sensations will interfere with one another [11].

\section{Haptic Feedback System Design}

A schematic and block diagram of the haptic feedback system as applied to the drivetrain control system can be seen in Fig. 2 . Temperature and current sensing circuits measure the temperature and current draw of both the left and right drivetrain motors. This information is read into the Robot Controller (RC), running the control program, which then transmits and receives information via radio modem to the Operator Interface (OI). The OI outputs control the vibration motors and heating coils through an electronic speed controller and relays, respectively, to provide feedback to the operator. 


\subsection{Onboard Sensors}

On the combot, a temperature sensing circuit measures the temperature of the exposed can of one of the drivetrain motors on each side. This temperature sensing circuit is a simple voltage divider consisting of a $10 \mathrm{k} \Omega$ resistor in series with a $10 \mathrm{k} \Omega$ negative temperature coefficient thermistor (Epcos Inc. Part \#B57871S0103F002). This thermistor has an operating range of $-55^{\circ} \mathrm{C}$ to $155^{\circ} \mathrm{C}$, above the $130^{\circ} \mathrm{C}$ limit of the electric motors. The resistor end of this series circuit was connected to ground and the thermistor end was connected to the $5 \mathrm{~V}$ supply of the RC. The junction between the resistor and thermistor was connected to one of the signal lines of the 8-bit analog inputs of the RC.

A hall effect current sensor (Allegro Microsystems, Inc. Part \# ACS758ECB-200B-PFF-T) was wired in series with one of the two pairs of drive motors on each side of the drivetrain to measure the current draw. With a sensitivity of $10 \mathrm{mV} / \mathrm{A}$, this sensor internally generates a 0 to $5 \mathrm{~V}$ signal in response to the current through its power tabs. A value of $2.5 \mathrm{~V}$ corresponds to no current draw, while 0 and $5 \mathrm{~V}$ correspond to currents of $\pm 250 \mathrm{~A}$, respectively. The signal from the current sensor was again read as an 8-bit analog input on the RC. Then, for a reference value, the maximum current draw was measured at stall to be $130 \mathrm{~A}$, well within the $250 \mathrm{~A}$ limit of the sensor. The physical layout of the motor, temperature sensor, and current sensor can be seen in Fig. 3. Though there are four drive motors on each side, they are identical and all linked to the same output gear; it was therefore expected that their current draw and heating would be nearly identical among all connected motors.

\subsection{Haptic Display}

The OI's primary function is to read the joystick and switch values for control of the combot and only has a limited ability to display feedback from the combot to the operator. This feedback consists of eleven LEDs displayed on the top of the OI, and eight LED output signals, originally intended to illuminate LEDs on a control box attached to the OI. We made use of four of these LED drivers to control the vibrotactile and thermotactile feedback, two for each side. It should be noted that the refresh rate of these drivers is limited by the communications cycle frequency between the OI and $\mathrm{RC}$, which is $38 \mathrm{~Hz}$ [5].

Two Innovation First Spike Relays, one for each side, were used to control the heating coils for thermotactile feedback. These relays were chosen for their current handling ability. These relays require a $0 \mathrm{~V}$ signal corresponding to off or a $5 \mathrm{~V}$ signal corresponding to on, as the OI naturally displays on the LED pin outs. Each relay, in turn, supplied power to two $1 \Omega, 10 \mathrm{~W}$, power resistors wired in series on each joystick handle. Over the three minutes of the match, the temperature of the motors does not decrease significantly so the heat accumulates, thus only single direction heating is needed in the thermotactile feedback. Therefore, simple resistive heating was used as opposed to peltier devices, which can both heat and cool but are relatively energy inefficient. The contact area of each set of heating coils was approximately $1.5 \mathrm{in}^{2}\left(10 \mathrm{~cm}^{2}\right)$. A temperature sensing circuit, like those used on the drivetrain motors, monitored the temperature of the heating coils. The signal from these temperature sensors were read into the 8 bit analog inputs on the OI, and used in a feedback loop to match the temperature of the heating coils to those of the motors. The response time of the heating coils was faster than that of motors.

A Sabertooth $2 \times 5$ electronic speed controller, in analog control mode, was used to control the vibrotactile motors. This speed controller was chosen because it has two channels, one for the current feedback for each side of the combot. For vibrotactile feedback, only the forward control is required. At $5 \mathrm{~V}$ input, the speed controller supplies the full battery voltage to the motor continuously, while at values between $2.5 \mathrm{~V}$ (no output) and $5 \mathrm{~V}$ the controller uses pulse width modulation to reduce the average

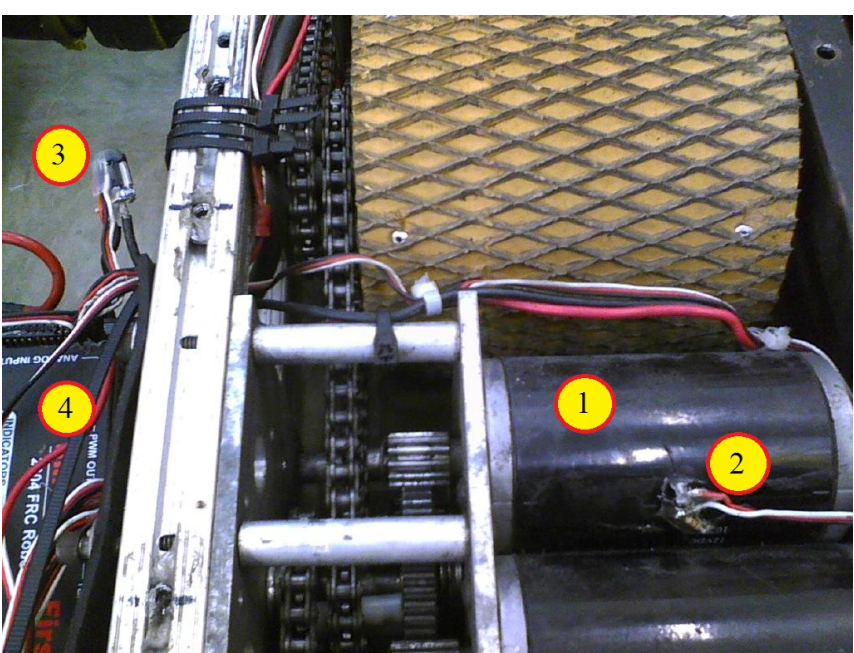

Figure 3: Physical layout of onboard sensors: (1) Drivetrain motor, (2) Temperature Sensor, (3) Current Sensor, (4) Robot Controller

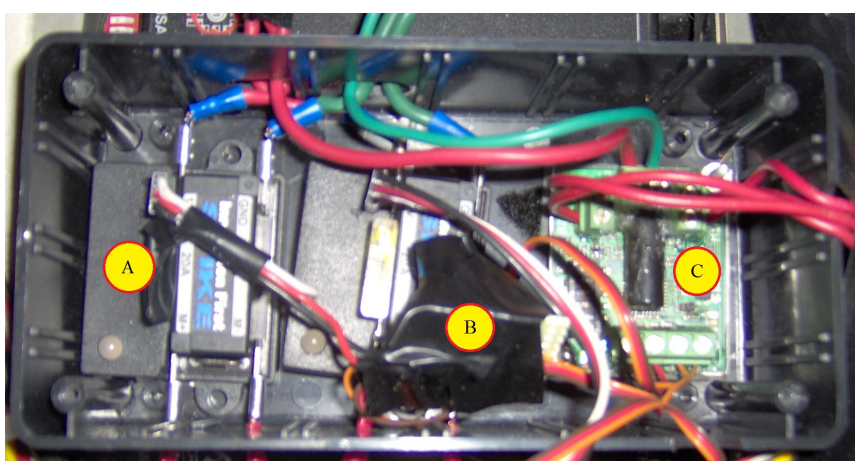

Figure 4: Haptic Feedback Control Box: (A) Thermotactile Relay, (B) Signal Conditioner, (C) Vibrotactile Speed Controller

voltage to the motor. A custom circuit was built to condition the signal from the LED driver so that when the LED signal was disabled the sabertooth received the $2.5 \mathrm{~V}$ neutral input signal, and when the $5 \mathrm{~V}$ LED was activated the sabertooth received a $5 \mathrm{~V}$ full forward input signal. With a time constant of $100 \mathrm{~ms}$, the signal conditioner also acted to smooth the voltage supplied to the speed controller and, therefore, the voltage to the vibrotactile motors. There was one signal conditioner for each of the two vibrotactile channels. The haptic feedback controllers were housed in a small electronics box as shown in Fig. 4.

The vibrotactile motor consisted of a $25 \mathrm{~mm}$ diameter RF-370 motor with an unbalanced flywheel pressed onto the motor shaft. Both the vibrotactile motors and the heating coils were attached to the left and right joysticks by vinyl tape as shown in Fig. 5. The heating coils are located under the operator's palm, while the vibrotactile motors were placed out of the way on the inner side of the joysticks. Power to the OI, both vibrotactile motors, and heating coils was supplied by an $11.1 \mathrm{~V}, 1300 \mathrm{mAh}$ lithium polymer battery. Testing showed this battery to last 5-10 minutes depending on the demands of the system, which is adequate for the 3 minute match length.

\subsection{Feedback Control}

If the value of the handle temperature is less than the value of the motor temperature, the heating coils are energized, otherwise they 


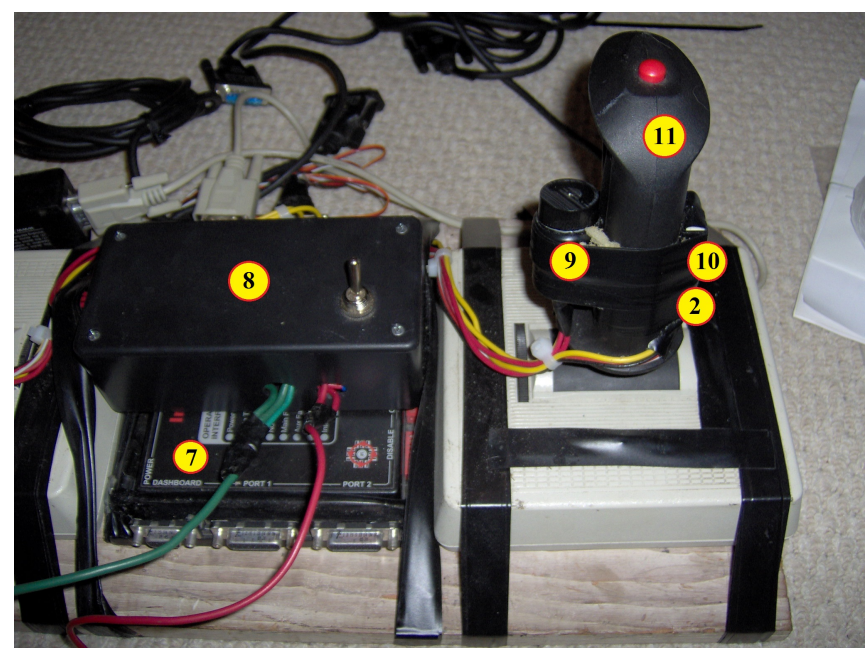

Figure 5: Operator Interface and Haptic Display: (2) Temperature Sensing Circuit, (7) Operator Interface, (8) Haptic Feedback Control Box, (9) Vibrotactile Motor, (10) Heating coils, (11) Control Joystick

are switched off. When the coils make a switch from off to on, or on to off, the program starts a timer, and waits for one second before switching again. This delay prevents rapid on-off switching of the mechanical relays when the handle and motor temperature values are the same, though it does increase overshoot when trying to match the temperature. The maximum temperature rendered by the heating coils is limited to $75^{\circ} \mathrm{C}$.

If the current draw is less than $2 \mathrm{~A}$ in either direction, then no voltage is applied to the vibrotactile motor. For nonzero values of current draw, the vibrotactile motor is switched on for one $26 \mathrm{~ms}$ cycle of the control system and then off for a number of cycles inversely related to the current draw by the following equation:

$$
\text { delay }=-11 \frac{|I|}{I_{\max }}+12
$$

where delay is the number of off cycles between on pulses, rounded up to the nearest integer, $I$ is the current draw, and $I_{\max }$ is the value of current measured at motor stall. The delay between on pulses therefore ranges between $312 \mathrm{~ms}$ at minimal load to $26 \mathrm{~ms}$ at stall. The result is a gentle pulsed vibration of the joystick at low current draw which increases in strength to a steady vibration similar to that of a typical cell phone vibration at maximum current draw. In testing, it was verified that the vibration frequency remained below the natural frequency of the system, and that the range of current draws and their corresponding vibrations were easily perceivable.

\section{Results ANd Discussion}

The device was initially tested by the first author (the primary driver of the combot) and a second participant with no experience with the combot. This initial testing consisted of simply driving the combot, freestyle, in an open area with no obstacles. The author reports that the vibrotactile current draw was easy to feel when actively trying to follow it. When the author was focused on the motions of driving, however, it became more difficult to monitor the current draw. The second participant reported a similar feeling of losing track of the vibrotactile feedback when operating the combot. The temperature of the drive motors only slightly increased during the initial testing, and this rise was not actively observed during the testing. At the end of this testing, it was verified that the motor and handle temperature were qualitatively similar.
Subsequently, the feedback device was tested at the 2011 Robogames, an international competition for a variety of robotic challenges. The haptic feedback proved useful in the first three minute long match of the tournament against an opponent with a powerful horizontal cutting weapon. For the majority of the match, the author did not perceive either of the feedback modes as he was preoccupied by attempting to outmaneuver the opponent and prevent the weapon from striking the weaker sides of the combot. After approximately 30 seconds, the author's combot was able to damage the opponent's weapon system, disabling its weapon. The match then became a matter of maintaining control until time expired. With approximately 45 seconds left in the match, the author noticed a slowing of the drivetrain and concluded the battery voltage was dropping, for the remainder of the match, the author used the vibrotactile current feedback to minimize current draw of the drivetrain, allowing the combot to maintain power through the end of the match to a victory.

Unfortunately, after the first match, it was noted that the voltage of the Operator Interface battery had dropped below a safe level, and it was decided to not enable the feedback system again until two battery packs could be placed in parallel for the OI. Damage to the combot in the second match forced the author to disconnect the sensors to make repairs to the drivetrain. Due to time required to make the feedback system functional again, it was disabled for the remainder of the tournament.

\section{CONCLUSIONS AND FUTURE WORK}

Though the testing data thus far has been limited, the haptic feedback device presented in this paper has shown promise as a method of rendering information on temperature and current draw of the electric motors on a combot. This information proved useful in reducing motor current draw in the match the device was tested in. Though the thermotactile temperature feedback proved less beneficial than vibrotactile current feedback, future improvements to this system could be made to improve it. These improvements include the installation of temperature sensors inside the motors to accurately measure the internal motor temperature and the use of solid state relays to eliminate noise from switching.

In addition to reducing current draw, this system could also be used to quickly diagnose damage at a distance. For example, if half of the drivetrain was acting sluggish, it would be possible to distinguish between a break in the power transmission system (low current draw), or something binding in the power transmission system (high current draw). This is important because the best actions to get through the rest of the match are very different for these scenarios, gentle acceleration to prevent more damage in the case of a break, or hard acceleration and direction changes to dislodge the object binding the system. For these reasons, we believe vibrotactile current feedback will be important in future combots. Future studies will investigate the ability of this feedback modality to affect fighting behavior.

This system could be improved by using a dedicated sensor and feedback system, isolated from the combot control system. This would reduce the refresh rate limitation of the OI, and allow for easier data logging. Separating the feedback and control system would also reduce the risk of physical damage to the sensors resulting in damage to the control system.

Additionally, because of its ease of implementation, we believe this system could be adapted for use on rescue or military telerobots where the torque on the telerobot's drive system could provide valuable information about the terrain that it is on and any obstacles that it may encounter.

\section{ACKNOWLEDGEMENTS}

The authors thank Southern Manufacturing Technologies for their financial support. 


\section{REFERENCES}

[1] D. Caldwell and C. Gosney. Enhanced tactile feedback (tele-taction) using a multi-functional sensory system. In Robotics and Automation, 1993. Proceedings., 1993 IEEE International Conference on, pages 955 -960 vol.1, may 1993.

[2] T. Debus, T. Becker, P. Dupont, T.-J. Jang, and R. Howe. Multichannel vibrotactile display for sensory substitution during teleoperation. In Telemanipulator and Telepresence Technologies VIII Conference, Proceedings of the SPIE, 2001.

[3] P. E. Devens and S. C. York. Battlebots laboratory an integrated team-based design/build course. In 2003 ASEE Southeastern Section Conference, 2003.

[4] J. Dong and J. Dave. Experience of designing and manufacturing a battlebot to compete. ASME Conference Proceedings, 2009(43802):335-340, 2009.

[5] Innovation First Inc. 2004 Programming Reference Guide, April 2004

[6] L. Jones and H.-N. Ho. Warm or cool, large or small? The challenge of thermal displays. Haptics, IEEE Transactions on, 1(1):53-70, Jan.June 2008.

[7] M. J. Massimino. Improved force perception through sensory substitution. Control Engineering Practice, 3(2):215 - 222, 1995.

[8] M. A. Meggiolaro. Riobotz Combat Robot Tutorial. CreateSpace, 2009.

[9] M. A. Meggiolaro. Riobotz Combot Tutorial Version 2.0, March 2009. http://www.riobotz.com.br/en/tutorial.html.

[10] B. E. Mullins and B. S. Peterson. Battlebots and the electrical engineering education. In Proceedings of the 2002 American Society for Engineering Education Annual Conference \& Exposition, 2002.

[11] G. Paxinos and J. Mai, editors. The human nervous system, chapter 28: Somatosensory Systems by Jon H. Kaas. Elsevier Academic Press London, 2004.

[12] C. Pylatiuk, A. Kargov, and S. Schulz. Design and evaluation of a lowcost force feedback system for myoelectric prosthetic hands. Journal of Prosthetics and Orthotics, 18(2):57-61, 2006.

[13] J. Smentowsky. Robot combat history by the Robot Marketplace, November 2002. http://www.robotcombat.com/history.html. 